\title{
Intelligent Monitoring Using Wireless Sensor Networks
}

\author{
Senol Zafer Erdogan ${ }^{1}$, Sajid Hussain ${ }^{2}$, and Jong-Hyuk Park ${ }^{3}$ \\ ${ }^{1}$ Faculty of Engineering, Maltepe University, Istanbul, Turkey \\ senole@maltepe.edu.tr \\ 2 Jodrey School of Computer Science, Acadia University, Wolfville, Canada \\ Sajid.Hussain@acadiau.ca \\ ${ }^{3}$ Department of Computer Engineering, Kyungnam University, Masan, Korea \\ parkjonghyuk@gmail.com
}

\begin{abstract}
Wireless sensor networks (WSNs) enable smart environments to provide pervasive and ubiquitous applications, which give contextaware and scalable services to the end users. In this paper, an agent-based architecture is proposed for knowledge discovery and the variation in received signal strength indicator (RSSI) is used for knowledge extraction. Several experiments are conducted in an in-door environment to demonstrate the application of RSSI for ubiquitous monitoring. For instance, a WSN, which consists of Moteiv's Tmote Sky sensors, is deployed in a bedroom to determine the sleeping behavior and other physical activities of a person. Similarly, a WSN is used to identify the occupied chairs in a room, as well as the mobility of a person.
\end{abstract}

\section{Introduction}

A smart environment can be created by using dense deployment of sensors to provide in-situ and precise monitoring, as well as using actuators to enable realtime, adaptive, and context-aware control mechanisms.

WSNs consist of a large number of smart sensors that have limited computing, storage, communication, and energy resources. These smart devices can interact with one another to create self-organized, ad hoc, and scalable networks that can provide intelligent, pervasive, and ubiquitous applications [1. For example, sensors networks are used in military, security, health-care [2], environment and habitat monitoring. Further, as computing, storage, and communication resources are very limited for current available sensors, there is a need for energy efficient algorithms and techniques to provide scalable solutions 3 .

As RSSI values vary because of mobility and obstruction, the RSSI variation can be used in the investigation of localization and mobility. Further, as the variation in RSSI values is non-uniform and non-deterministic with respect to distance and time, the simulation studies are not sufficient. As a result, the deployment environment should be investigated with real WSN experiments. In this paper, several experiments are conducted using Moteiv's Tmote Sky sensors to study the behavior of RSSI values for localization and mobility of a person in an in-door environment. The experiment results confirm that the variation 
in RSSI values can be used for localization and mobility. For example, sensors deployed in a bed room are used to determine the following: a) time spent at the study table, b) time spent on a bed, and c) wake-up time. Similarly, in a classroom environment, RSSI values are used to identify the occupied chairs, as well as the duration the chairs were occupied.

The remaining paper is organized as follow: Section 2 gives a brief description regarding RSSI related research. Section 3.1 describes an architecture for a smart application. Section 4 provides the experiment details and results. Finally, Section 5 concludes the paper.

\section{Related Work}

There is an active research in estimating the values of RSSI, link quality indicator (LQI), and packet reception rate (PRR) for realistic radio communication 4, [5], 6], 7], 8]. Zhou et al. [4] investigate the degree of variation in RSSI values and propose a non-circular radio irregularity model (RIM) for sensor networks. Gallais et al. 9 discuss the effect of a realistic radio channel on area coverage protocols. Scott et al. [10] use transmit and receive signal strengths to investigate propagation patterns. Halkidi et al. [1] use an online mechanism to determine the overall network status and to reduce the communications costs.

Erdogan and Hussain [12 investigate radio irregularity with respect to distance, sending power level, direction, and alignment of the sensor node from the base station. The experimental results show that proper alignment and sending power level can reduce the energy consumption, in order to increase the network lifetime. In this paper, however, the focus is not on reduced energy consumption but the objective is to extract knowledge and context information from the RSSI values. The variance in RSSI values can be used to identify user behavior, mobility, and environment.

RFID tags are commonly used for precise object tracking and inventory control [13] 14; however, these tags could be inconvenient for general human usage because RFID tags must be attached to a person. Senor motes, on the other hand, can be used to detect mobility or user behavior without any wiring or a tag attached to a person. Certainly, the sensor motes cannot provide the exact identification accuracy as of RFID tags; however, in several applications, we do not need the exact identification. The sensor motes can provide unobtrusive monitoring at common places such as hospitals, restaurants, homes, schools, and offices. Further, the sensor motes can also help in maintaining person's privacy. Although the information about the behavior of a person would be known, it would be relatively easy to hide the person's identity.

\section{Architecture}

\subsection{Terms and Definitions}

As the RSSI values vary with respect to the deployment environment, we describe a few terms that would be needed in the investigation of RSSI. 


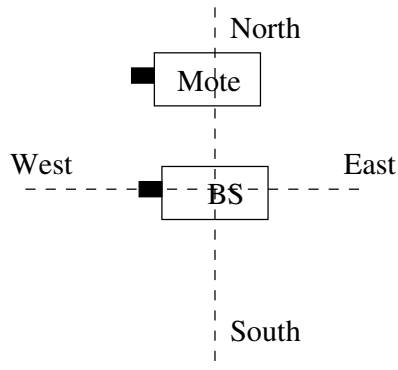

(a) North $(\theta=0)$

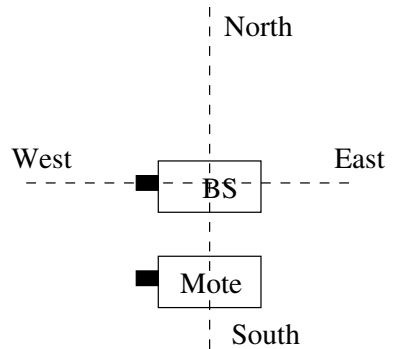

(c) South $(\theta=180)$

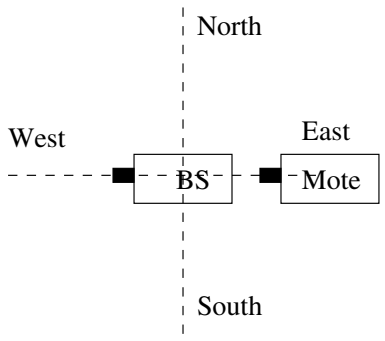

(b) East $(\theta=90)$

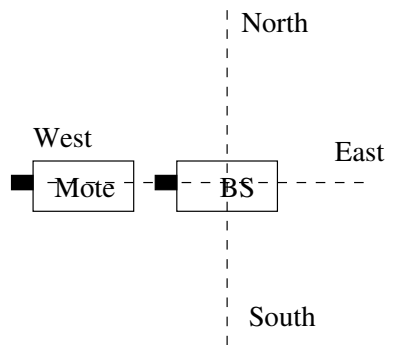

(d) West $(\theta=270)$

Fig. 1. Directions for node placements

Definition 1. Direction $\theta$ is defined as the angle with respect to the geographical direction, where North, East, South, and West are considered as 0, 90, 180, and 270 respectively.

Direction is used to identify the physical location of a node with respect to a given node. Figure 1 illustrates some of the examples where a node can be placed. For instance, Figure 1(a) shows that a node is placed at North direction $(\theta=0)$ with respect to the base station. Similarly, Figure 1(b), Figure 1(c) and Figure $1(\mathrm{~d})$ show that nodes are placed at East, South, and West directions respectively. Further, we can use angle to identify the location. The angle starts from North direction and continues in clockwise direction. For example, the directions of nodes placed at North, East, South, and West can be represented as $\theta=0, \theta=90, \theta=180$, and $\theta=270$.

Definition 2. Alignment $\gamma$ is defined as the angle between two motes.

Figure 2 shows different alignments for a sensor mote. Figure 2(a) and Figure 2(b) show sensor motes that are in North $(\theta=0)$ direction but alignments are $\gamma=0$ and $\gamma=90$ respectively. However, motes in Figure 2(b) and Figure 2(c) have same alignment $(\gamma=90)$ but their directions are North $(\theta=0)$ and East $(\theta=90)$ respectively.

Definition 3. Distance $d$ is as defined as a Euclidean distance between two motes. 


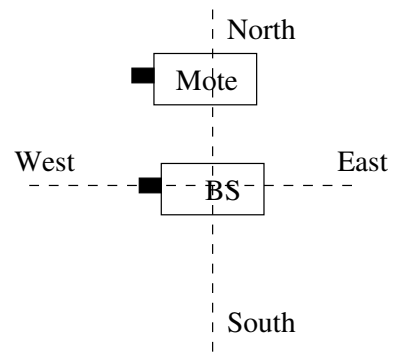

(a) $\theta=0, \gamma=0$

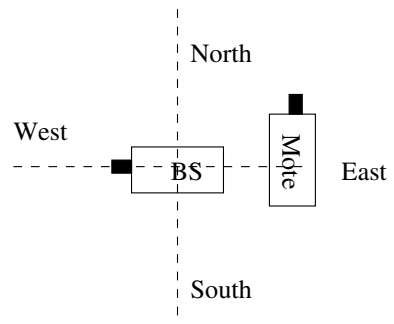

(c) $\theta=90, \gamma=90$

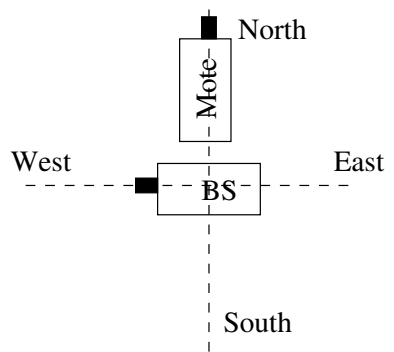

(b) $\theta=0, \gamma=90$

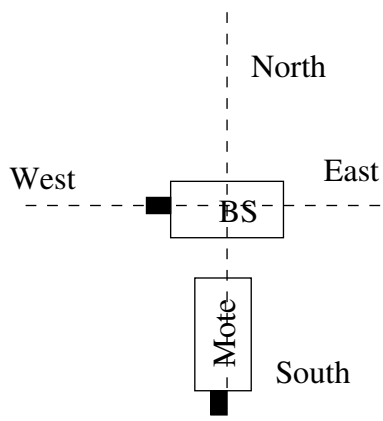

(d) $\theta=180, \gamma=270$

Fig. 2. Alignment of sensor motes

The distance $d$ can be measured directly between two motes or can be computed from their co-ordinates, as shown below:

$$
d=\left|\sqrt{\left|y_{2}-y_{1}\right|^{2}+\left|x_{2}-x_{1}\right|^{2}}\right|
$$

Distance is commonly used in localization techniques based on RSSI estimation; however, the distance $d$ parameter should be combined with direction $\theta$ and alignment $\gamma$ parameters for accurate investigation of deployment environment. For instance, in many radio communication models, energy consumption is considered as directly proportional to the square of distance (circular model) [15], which is not applicable in most of the realistic environments. As a result, change in RSSI $(\triangle R S S I)$ is a function of the above parameters:

$$
\Delta R S S I=f(\theta, \gamma, d)
$$

Further, the above equation is valid for static environment only. For dynamic or mobile environments, RSSI will also vary with respect to time, as given below:

$$
\Delta R S S I=f(\theta, \gamma, d, t)
$$

For instance, if sensors are mobile or if there are other moving objects in the neighborhood, RSSI will vary with respect to time, as given in Equation 3. 
An architecture for a WSN application to provide knowledge discovery and data mining would contain the following:

- A base station (BS) to connect the sensor network to traditional networks.

- A service agent that would contain the following entities: a) a communication component to abstract the communication details of the application, b) database component to store the data retrieved from the WSN, c) knowledge component to extract knowledge from the data stored in a database, and d) context-aware services for the application users. As service agent needs extensive computing and storage resources, it must be located on a computer or a gateway node.

- Sensor nodes to provide in-situ monitoring.

- WSN agents would be located at sensor nodes. Although the WSN agents would have similar components as a service agent; the WSN agent's components would provide minimal functionality because of limited resources of the sensor.

- An application (say Web application, Web App) to provide customized services to the end users.

Figure 3 shows the proposed agent-based architecture for the knowledge discovery application. The base station acts as a bridge between sensor network and the regular network. The application contains a service agent that can provide several context-aware services. The service agent contains the following entities: a communication component, database, and a knowledge extraction component. The communication component interacts with the external entities, such as base station. The communication component is connected to both knowledge component and database. All the incoming data is directly stored in a database for offline processing and stable storage. Further, the communication component is directly connected to the knowledge component, in order to provide real-time analysis and services. The service agent provides a given number of contextaware services to the subscribed users or applications. A web application, for instance, can use these context-aware services to provide customized web pages to the end user. Moreover, each sensor contains a sensor agent that has features

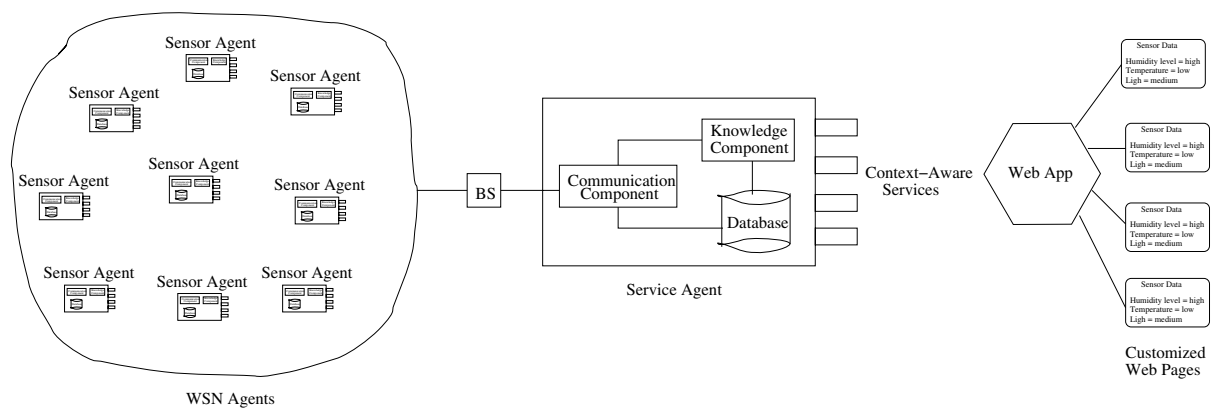

Fig. 3. Agent-based Architecture for Smart Applications 
identical to service agent; however, the sensor agent has minimal functionality because of limited computing and energy resources of sensors.

Service agent's knowledge component extracts non-trivial information from the incoming data stream. For instance, for a WSN deployed in a bedroom environment, the variation in the variance of RSSI values can be used to identify the sleeping behavior, study habits, and physical activities of a person.

\section{Experiment Results}

Experiments are conducted in an in-door environment to investigate localization and mobility of a person. The variance in RSSI values is used to identify the current location and activities of a person.

\subsection{Localization Example}

Figure 4 shows the experimental setup for a localization example. The sensors are deployed on 4 tables to identify any activity near the tables. The variation in RSSI values is used to determine the mobility or activity near a table. The experiment details are as follows:

- Base station is in the center of the room.

- There are 4 pairs of table and chair in four corners of the room. The distance between horizontal (along $\mathrm{X}$-axis) tables is $7.8 \mathrm{~m}$ and the distance between vertical (along Y-axis) tables is $5 \mathrm{~m}$.

- Moteiv's Tmote Sky 1 sensors are used for the base station and the 4 sensor motes.

- The time span for one experiment is 7 minutes. For confidence, the same experiment is conducted for 3 times.

- One person alternately sits on all the chairs.

The sensor motes send a packet to the base station after every second. For an occupied chair, the variance in RSSI values will be significantly different as compared to unoccupied chairs. As a result, the RSSI variance is used to identify the location and mobility of a person.

Figure 5 shows the RSSI values for 7 minute time interval (approximate) for all motes. Figure 5(a) shows RSSI values for a mote that is near Chair 1. Since Chair 1 is occupied during 1-65 seconds, there is relatively high variation in RSSI values. In other words, the high variation can determine the chair occupancy. However, during the same time interval, the RSSI values for mote 2, mote 3 and mote 4 are almost constant. Thus, it can be assumed that unoccupied chairs have relatively low variation in RSSI values. Similarly, Figure 5(b) Figure 5(c), and Figure 5(d) show the RSSI values for motes near Chair 2, Chair 3, and Chair 4 respectively. As expected, for all motes, the variation in RSSI values is relatively high for occupied time interval as compared to unoccupied duration, when the chair is unoccupied. The above experiment determines the following:

\footnotetext{
${ }^{1}$ http://www.moteiv.com
} 


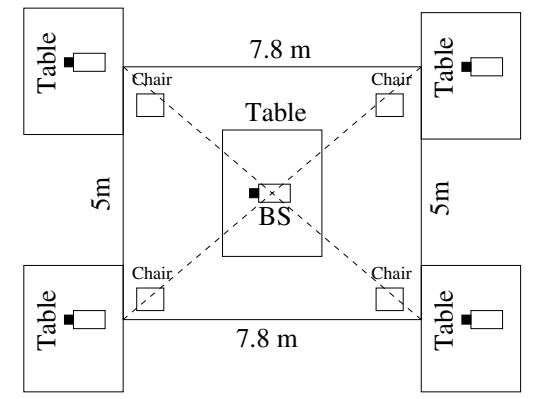

Fig. 4. Room Layout for the Localization Experiment

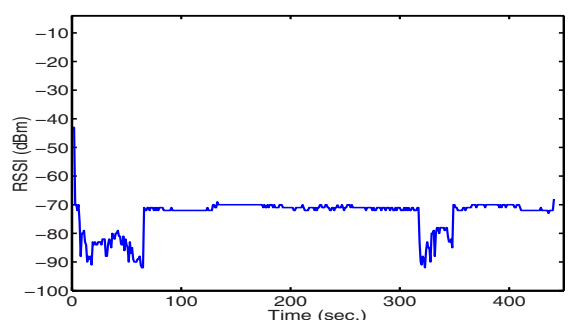

(a) Mote 1

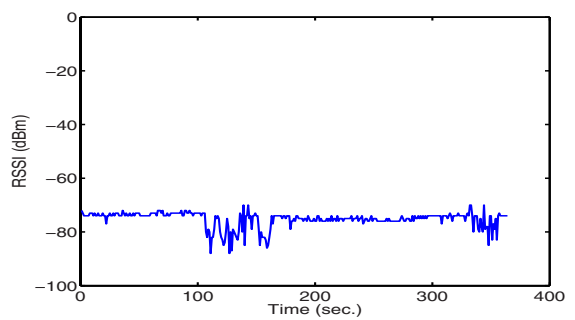

(c) Mote 3

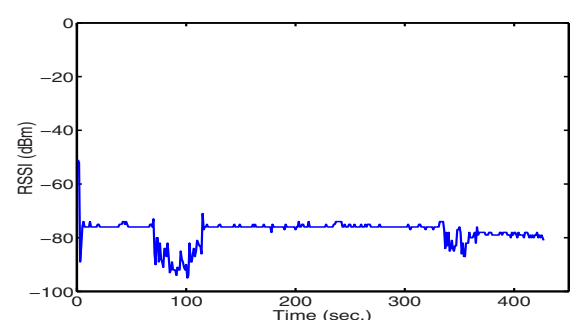

(b) Mote 2

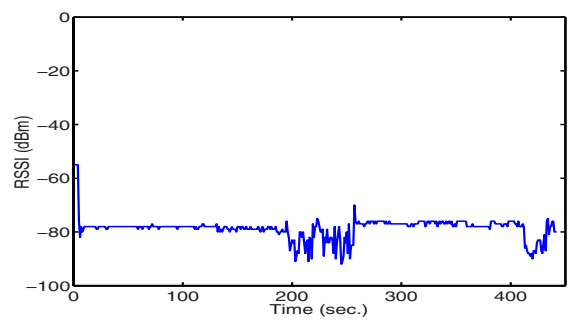

(d) Mote 4

Fig. 5. RSSI variation for 4 motes

- Each chair was occupied for a some period of time.

- At one time, only one chair was occupied.

- There is a rapid (or spontaneous) activity in moving from one chair to the next.

Table 1 shows the variance in RSSI values for different time intervals. As three experiments are conducted, the variance of each experiment is given for each mote. The results confirm the observation that variance in RSSI values can determine the occupied chair. For instance, for time interval 1-65 seconds, when chair 1 is occupied, the variance in Mote 1 is significantly high as compared to the remaining time intervals when the Mote 1 was unoccupied. Similarly, variation 
Table 1. Variance in RSSI values for Motes

\begin{tabular}{|c|c|c|c||c|c|c||c|c|c||c||c|c|}
\hline \multirow{2}{*}{ Time (sec.). } & \multicolumn{9}{|c||}{ Variance in RSSI values } \\
\cline { 2 - 14 } & \multicolumn{3}{|c|}{ Mote 1 } & \multicolumn{3}{c||}{ Mote 2 } & \multicolumn{3}{c||}{ Mote 3 } & \multicolumn{3}{c|}{ Mote 4 } \\
\hline & Exp 1 & Exp 2 & Exp 3 & Exp 1 & Exp 2 & Exp 3 & Exp 1 & Exp 2 & Exp 3 & Exp 1 & Exp 2 & Exp 3 \\
\hline $1-65$ & $\mathbf{5 0 . 8}$ & $\mathbf{4 5 . 3}$ & $\mathbf{4 8 . 1}$ & 0.37 & 1.24 & 1.53 & 0.49 & 0.94 & 1.12 & 0.46 & 0.59 & 1.13 \\
\hline $66-130$ & 0.26 & 0.84 & 1.23 & $\mathbf{4 4 . 4 6}$ & $\mathbf{4 8 . 6 3}$ & $\mathbf{3 9 . 3 2}$ & 1.96 & 2.34 & 1.70 & 0.1 & 1.02 & 0.83 \\
\hline $131-190$ & 0.17 & 0.46 & 0.79 & 0.20 & 0.89 & 1.93 & $\mathbf{1 5 . 1 8}$ & $\mathbf{1 7 . 4 3}$ & $\mathbf{1 7 . 2 1}$ & 0.55 & 0.79 & 0.94 \\
\hline $191-260$ & 0.35 & 0.22 & 0.54 & 0.49 & 0.94 & 1.03 & 0.53 & 1.21 & 0.93 & $\mathbf{2 3 . 1 0}$ & $\mathbf{2 9 . 6 2}$ & $\mathbf{3 1 . 4 2}$ \\
\hline
\end{tabular}

in RSSI variances is high for motes 2,3 , and 4 when their corresponding chairs are occupied.

The above experiment can be used in the following applications:

- Industrial exhibition: popular information posters, displays, or demos can be determined.

- Restaurants: the unoccupied tables, as well as popular tables can be identified.

- Parking: the available parking spots.

In another experiment, two sets of tables and chairs are used. A person alternately sits on a chair for 5 minute time interval. The distance between two tables is $5 \mathrm{~m}$. Figure 6 shows variation in RSSI values for both motes. As shown in Figure 6(a) and Figure 6(b) the variation in RSSI values can determine the occupied chair. For instance, in time interval 1-5 minutes (300 seconds), table 1 is occupied and table 2 is unoccupied. However, in time interval 6-10 minutes (300 - 600 seconds), table 2 is occupied and table 1 is unoccupied.

The above results can be used to develop WSN-based localization and contextaware services. For instance, in a museum or an art gallery environment, the time spent by visitors on a specific master-piece can be determined by the above

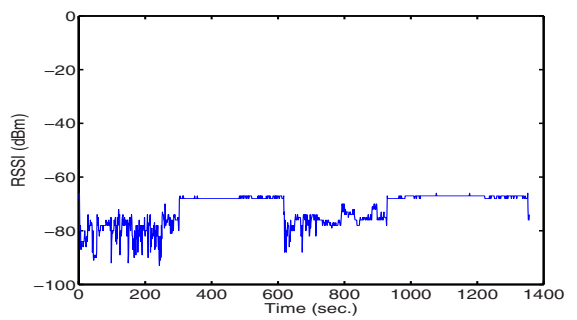

(a) Mote 1

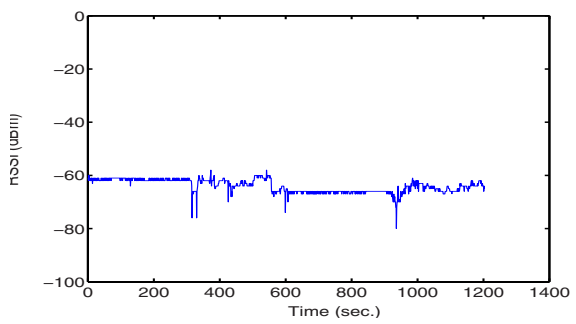

(b) Mote 2

Fig. 6. RSSI variation for 2 motes with 2 tables 
experiment. Similarly, the above experiments can also be used to identify the popular booths or demos in an industrial or art exhibition.

\subsection{Monitoring Human Behavior in a Bedroom}

In this experiment, sensors are deployed in a bedroom to monitor the behavior, mobility or lifestyle of a person. For instance, the sensors can be used to determine the time spent on the study table or on a bed.

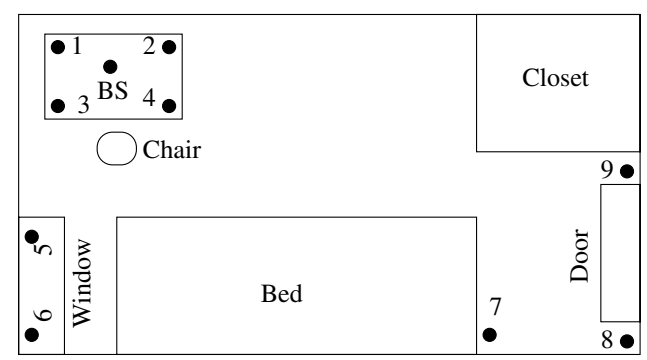

Fig. 7. Room plan of a bedroom

Figure 7 shows the room plan of a bedroom. The sensors are deployed as follows: 4 sensors on a study table (Mote 1, Mote 2, Mote 3, and Mote 4), 2 sensors near the window (Mote 5 and Mote 6), 1 sensor on the corner of bed (Mote 7), and 2 sensors near the door (Mote 8 and Mote 9). The base station is attached to a laptop on the study table. The sensor data is collected for several days.

Figure 8 shows RSSI values for table, window and door sensors. RSSI variation confirms with the actual behavior of a person. For instance, as recorded by the person, the RSSI variation confirms the behavior observed by the sensors. The student was working at the study table for the initial 3 hours, which is confirmed by RSSI variation for Table motes 1 and 3, as shown in Figure 8(a) and Figure 8(c) respectively. Further, the small variation for Table motes 2 and 4 (Figure $8(\mathrm{~b})$ and Figure $8(\mathrm{~d})$ indicates that the person's sitting position did not affect these values. By comparing the RSSI values of Table motes 1, 2, 3, and 4 , the sitting posture or inclination on a table can also be determined, although it is not investigated in this paper.

After 3 hours of working, the person slept for 7-8 hours. The sleeping time is also evident from RSSI values of window and door motes. Further, the RSSI variation for door motes are not consistent. For instance, Mote 8 (Figure 8(g) near the bed confirms the sleep behavior; however, the mote near the closet (Mote 9) shows some unexpected RSSI variation. Finally, the RSSI variation in the last couple of hours shows the morning activity, which is confirmed by most of the motes. 


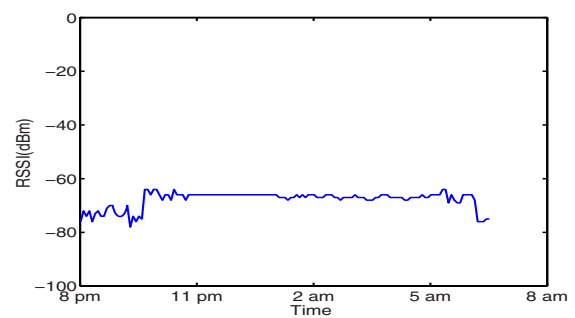

(a) Table: Mote 1

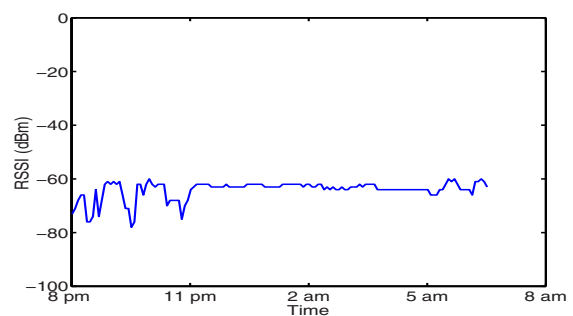

(c) Table: Mote 3

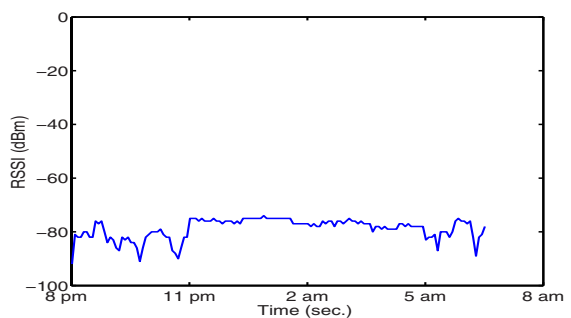

(e) Window: Mote 5

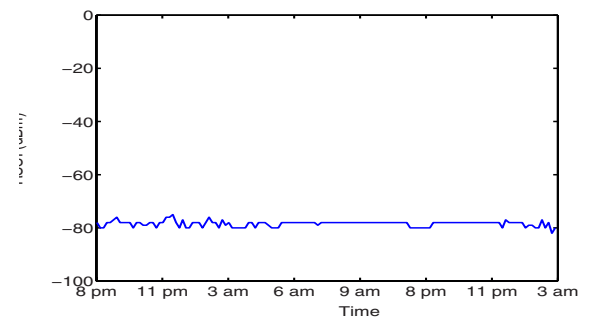

(g) Door: Mote 8

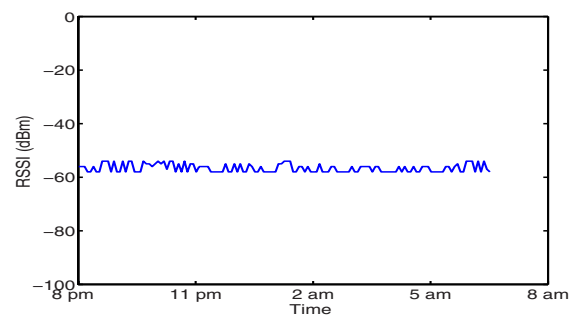

(b) Table: Mote 2

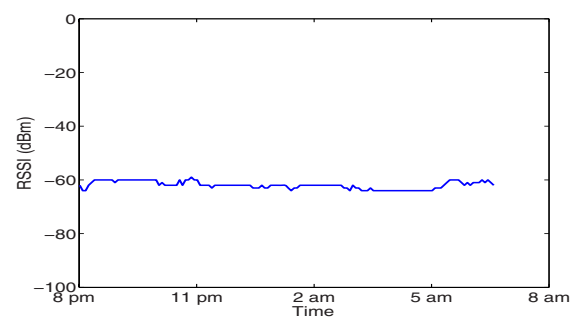

(d) Table: Mote 4

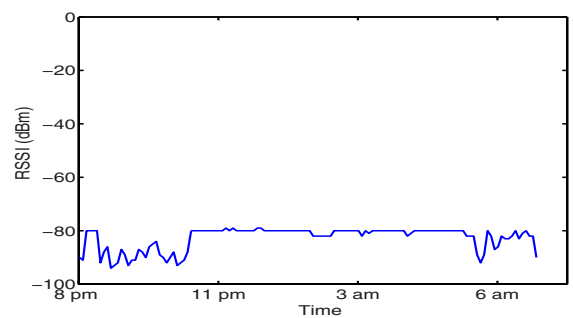

(f) Window: Mote 6

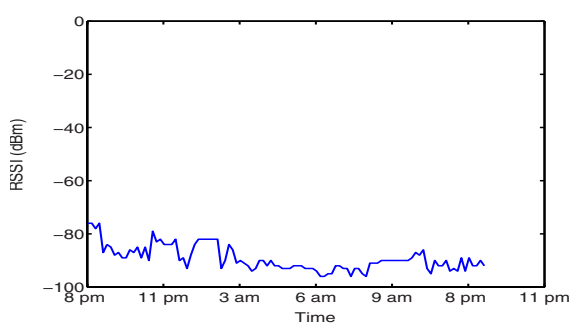

(h) Door: Mote 9

Fig. 8. RSSI variation for sensors in a bedroom

The above results can be used in maintaining a daily activity diary for a person. The results obtained at the base station can be logged in a database and can be retrieved through a web-based application. Consequently, a life style of a person, active or sedentary, can be estimated by these results. 


\section{Conclusion and Future Work}

In this paper, an agent-based architecture is proposed for knowledge discovery. The variation in RSSI values is used for knowledget extraction. Several experiments are conducted in an in-door environment such as: a) a WSN is deployed in a bedroom to determine the sleeping behavior and other physical activities of a person, b) a WSN is used to identify the occupied chairs in a room, as well as the mobility of a person, and c) humidity sensor of Moteiv's Tmote Sky is used for knowledge extraction.

In future, the fuzzy-based logic and other machine learning techniques will be used for knowledge discovery and data mining. Further, the context-aware services and end-user applications will be provided.

\section{References}

1. Akyildiz, I.F., Su, W., Sankarasubramaniam, Y., Cayirci, E.: A survey on wireless sensor networks. IEEE Communication Magazine (2002)

2. Gao, T., Greenspan, D., Welsh, M., Juang, R.R., Alm, A.: Vital signs monitoring and patient tracking over a wireless network. In: The 27th Annual International Conference of the IEEE EMBS, Shanghai, China (2005)

3. Kahn, J., Katz, R., Pister, K.: Next century challenges: Mobile networking for smart dust. In: MobiCom 1999. The ACM International Conference on Mobile Computing and Networking, Seattle, USA (1999)

4. Zhou, G., He, T., Krishnamurthy, S., Stankovic, J.A.: Impact of radio irregularity on wireless sensor networks. In: MobiSys. The International Conference on Mobile Systems, Applications, and Services, Boston, USA (2004)

5. Gu, L., Jia, D., Vicaire, P., Yan, T., Luo, L., Tirumala, A., Cao, Q., He, T., Stankovic, J.A., Abdelzaher, T., Krogh, B.H.: Lightweight detection and classification for wireless sensor networks in realistic environments. In: The 3rd ACM Conference on Embedded Networked Sensor Systems, San Diego, USA (2005)

6. Cerpa, A., Busek, N., Estrin, D.: Scale: A tool for simple connectivity assessment in lossy environments. In CENS Technical Report 0021 (2003)

7. Ganesan, D., Krishnamachari, B., Woo, A., Culler, D., Estrin, D., Wicker, S.: Impact of radio irregularity on wireless sensor networks. In Technical Report UCLA/CSD-TR 02-0013 (2002)

8. Woo, A., Tong, T., Culler, D.: Taming the underlying challenges of reliable multihop routing in sensor networks. In: SenSys 2003, Los Angeles (USA) (2003)

9. Gallais, A., Parvery, H., Carle, J., Gorce, J.-M., Simplot-Ryl, D.: Efficiency impairment of wireless sensor networks protocols under realistic physical layer conditions. In: ICCS 2006. 10th IEEE International Conference on Communication Systems, Singapore (2006)

10. Scott, T., Wu, K., Hoffman, D.: Radio propagation patterns in wireless sensor networks: New experimental results. In: IWCMC 2006. IEEE International Wireless Communications and Mobile Computing Conference, Vancouver, Canada (2006)

11. Halkidi, M., Kalogeraki, V., Gunopulos, D., Papadopoulos, D., Zeinalipour-Yazti, D., Vlachos, M.: Efficient online state tracking using sensor networks. In: MDM 2006. The 7th International Conference on Mobile Data Management (2006) 
12. Erdogan, S., Hussain, S.: Experiences in realistic radio communication forwireless sensor networks. Technical Report, Jodrey School of Computer Science, Acadia University, TR-2007-002 (2007)

13. Vogt, H.: Efficient object identification with passive rfid tags. LNCS. Springer, Heidelberg (2002)

14. De, P., Basu, K., Das, S.K.: An ubiquitous architectural framework and protocol for object tracking using rfid tags. In: MOBIQUITOUS. The First Annual International Conference on Mobile and Ubiquitous Systems: Networking and Services (2004)

15. Heinzelman, W.R., Chandrakasan, A., Balakrishnan, H.: Energy-efficient communication protocol for wireless microsensor networks. In: Proceedings of the Hawaii International Conference on System Sciences (2000) 